\title{
Optimal Transmission Scheduling in Small Multimodal Underwater Networks
}

\author{
Filippo Campagnaro, Paolo Casari, Senior Member, IEEE, \\ Michele Zorzi, Fellow, IEEE, Roee Diamant, Senior Member, IEEE
}

\begin{abstract}
We describe a scheduling protocol for multimodal networks of relatively limited size, whose nodes encompass various underwater communication technologies. For such a case, we show that significant improvement in the network operations is possible when the transmission schedule is set to jointly utilize all communication technologies. Our solution is based on per-technology TDMA frames, whose time slots are determined optimally to maximize the overall channel utilization while preserving flow limitations and maintaining fairness in resource allocation. Our numerical simulations and experimental results for multimodal networks with several acoustic technologies show that, while maintaining a fair resource allocation, our scheduling solution provides both high throughput and low packet delivery delay.
\end{abstract}

Index Terms-Underwater communication networks; underwater acoustic communications; multi-modal systems; transmission scheduling

\section{INTRODUCTION AND RELATED WORK}

Underwater communications have gradually become the enabler of several types of submerged operations. Submarines, divers, autonomous underwater vehicles (AUVs), and floaters are often endowed with underwater communication capabilities. This has progressively led to the formation of underwater networks, where devices share their measured data and act to collaborate with other sensors. The increase of communication systems and underwater operations will soon result in multimodal networks, where a node incorporates multiple physical layer (PHY) technologies, e.g., acoustic, optical and radio-frequency (RF). This will enable new applications, such as data muling and wireless telemetry for hybrid vehicles.

With several heterogeneous nodes, employing a different sub-network for each technology may result in disconnections and poor data transfer performance. Instead, it would be possible to increase the throughput, decrease the communication delay via simultaneous transmissions and reduce the occurrence of bottlenecks by properly leveraging the full set of PHYs. The main challenge, and the focus of this letter, is how to optimally schedule the transmission of the available PHYs.

Different from multi-band scheduling, which aims mostly at interference avoidance, multimodal networks

This work was sponsored in part by the NATO Science for Peace and Security Programme under grant G5293.

F. Campagnaro (email: campagn1@ dei.unipd.it) and M. Zorzi (email: zorzi@dei.unipd.it) are with the Department of Information Engineering, University of Padova, 35131 Padova, Italy. P. Casari (email: paolo.casari@imdea.org) is with IMDEA Networks, 28198 Madrid, Spain. R. Diamant (email: roeed@univ.haifa.ac.il) is with the Department of Marine Technologies, University of Haifa, Haifa 3498838, Israel. enable diversity in terms of the PHY itself. For example, low-frequency acoustic communications achieve kbit/s transmission rates over ranges of a few kilometers, highfrequency acoustics tops tens of kbit/s over up to a few hundred meters, whereas optical communications yield Mbit/s links over ranges of a few meters. Different PHYs face different challenges: for example, acoustic communications are sensitive to time-varying multipath, whereas water turbidity and ambient light hamper optical communications [1]. Hence, efficient multimodal networking requires a specific scheduling solution with different properties than the schemes in single-technology network domains.

The approaches designed for multi-radio or multichannel wireless radio networks rely on frequent communications or feedback, and target the management of voice calls rather than data transmission [2]. Scheduling poses different requirements in multimodal underwater networks, where the available PHYs have widely different communication capabilities, and the scheduler must account for PHY-dependent adjacency and interference matrices while avoiding bottlenecks.

For underwater multimodal operations, it has been proposed to use visual image processing [3] or signaling to identify the fastest available PHY [4]. The MURAO protocol [5] organizes the nodes in clusters, using optics for intra-cluster communications and acoustics for cluster management. The above approaches are tailored to specific scenarios, or offer solutions for stable networks. However, they may suffer from bottlenecks and delays in realistic multimodal networks, where PHY performance changes over time and space due to mobility and environmental conditions, and does so in different ways for different PHYs. A scheduling mechanism that can optimize the use of the multimodal network's resources is therefore needed.

To address the above challenges, we propose the optimal multimodal scheduling (OMS) protocol. OMS manages transmissions through any set of PHYs by jointly setting transmission time slots in a per-technology time-division multiple access (TDMA) fashion, and divides the data load among the PHYs to optimize link utilization and transmission delay. In addition, OMS organizes transmission slots to favor packet routing and enforce a fair number of transmission opportunities per node. We tested the performance of OMS against benchmark schemes in numerical simulations and in a sea experiment using multimodal nodes encompassing different acoustic PHYs. The results show that OMS achieves better throughput, packet delivery delay, and fairness in resource allocation. 


\section{THE OMS AlgorithM}

\section{A. System Model}

Our system consists of $N$ network nodes equipped with one or more of $T$ underwater PHYs. The set of PHYs is arranged in the $N \times T$ technology matrix $\mathbf{T}$ such that $\mathbf{T}_{i, n}=1$ if node $i$ has PHY $n$. Call $\mathbf{M}$ the adjacency matrix, where $\mathbf{M}_{i, j, n}=1$ if node $i$ is connected to node $j$ via PHY $n$. The number of neighbors of $i$ through technology $n$ is $\mathbf{D}_{i, n}=\sum_{j} \mathbf{M}_{i, j, n}$. We assume that $\mathbf{T}$ is given, and that $\mathbf{M}$ can be obtained via preliminary link probing [6]. We remark that the difference between the communication and interference range is limited in underwater networks, due to the very fast power decay incurred for increasing range by any PHY technology [4]. Hence, to harness spatial reuse for performance gain, we allow collisions in OMS.

OMS organizes orthogonal multimodal PHYs via pertechnology TDMA frames. As different PHYs are characterized by diverse transmission rates and may incur different propagation delays (e.g., optics vs. acoustics), the duration of the time slots is also set per-technology. We choose TDMA since it allows a simple time slot alignment via guard intervals. This is specifically important in multimodal systems, where different PHYs have diverse outage capacity. Additionally, in TDMA-based schemes the transmission delay is known in advance, making it possible to plan the load allocated to each PHY. This is in contrast to handshake-based schemes (where the delay depends also on the receiver) and to fully random access (where collisions may trigger an unpredictable number of retransmissions). The synchronization of the low latency technologies can be achieved either via atomic clocks or through the network time protocol (NTP). For acoustics, however, we can simply rely on guard times: as the time slot duration is at least as long as the maximum propagation delay, such guard times are negligible.

We impose traffic constraints by allowing a node $i$ to transmit in at least $c_{i}>C$ time slots. Calling $R_{i}$ the number of bits transmitted in each time slot of the slowest communication technology of node $i$, constraint $c_{i}$ ensures the transmission of at least $c_{i} R_{i}$ bits within a given frame. Fairness then results from setting $c_{i}$ such that nodes with lower $R_{i}$ receive a higher $c_{i}$ value.

\section{B. OMS Scheduling Solution}

Our solution allocates transmission time slots, organized in $n$ TDMA frames of $N$ slots and duration $\tau_{n}^{\text {sl }}$ : one frame for each PHY. We synchronize transmissions by considering a TDMA super-frame of length $\tau^{\text {fr }}$, such that for some PHYs several (not necessarily full) TDMA cycles are possible per $\tau^{\mathrm{fr}} \mathrm{s}$. The input to OMS is the PHY matrix $\mathbf{T}$, the adjacency matrix $\mathbf{M}$, the per-PHY communication capacity, and the number of slots $N$ and time slot duration $\tau_{n}^{\mathrm{sl}}$. The output is the minimum allowed value of $\tau^{\mathrm{fr}}$, and a matrix $\mathbf{S}$, where $\mathbf{S}_{i, t, n}=1$ if node $i$ can transmit in slot $t$ via PHY $n$. The transmission slot indices are arranged in a vector $\mathbf{t}_{i, n}^{\mathrm{Tx}}=\left\{r: \mathbf{S}_{i, r, n}=1\right\}$ for node $i$ over technology $n$. Our objective is to maximize channel utilization, measured via the total number of transmissions over a given time period. The schedule also considers collisions among neighboring nodes and facilitates the forwarding of packets across multiple hops. OMS requires the knowledge of the adjacency matrix $\mathbf{M}$ obtained, e.g., via [6]. This includes the existing connections and the available per-node PHY technologies.

Let $\vee$ be the logical "or" and $\wedge$ the logical "and" operators. The optimal schedule $\mathbf{S}^{\star}$ with time frame length $\tau^{\mathrm{fr}}$ is the solution of the following problem:

$$
\begin{aligned}
& \mathbf{S}^{\star}, \widehat{\tau^{\mathrm{fr}}}=\underset{\tau^{\mathrm{fr}}}{\arg \min }\left(\max \sum_{i} \sum_{t} \sum_{n} c_{i} \mathbf{S}_{i, t, n}\right) \\
& \text { s.t. } \mathbf{T}_{i, n}=0 \Longrightarrow \mathbf{S}_{i, t, n}=0 \\
& \mathbf{S}_{i, t, n}=1 \wedge \mathbf{S}_{j, t, n}=1 \Longrightarrow \\
& \left(\mathbf{M}_{i, p, n}+\mathbf{M}_{j, p, n}=0\right) \\
& \vee\left(\mathbf{M}_{i, p, n}+\mathbf{M}_{j, p, n}=1 \wedge \mathbf{S}_{p, t, n}=0\right) \\
& \vee\left(\mathbf{M}_{i, p, n}+\mathbf{M}_{j, p, n}=2 \wedge \mathbf{S}_{p, t, n}=1\right) \forall p \neq i, j \\
& \sum_{t} \sum_{n} \frac{\mathbf{S}_{i, t, n}}{\mathbf{D}_{i, n}} \geq c_{i} \geq C \quad \forall i, n \\
& \exists t \text { s.t. } \mathbf{S}_{i, t, n}=1, \mathbf{S}_{j, t, n}=0 \forall i, j, n \text { s.t. } \mathbf{M}_{i, j, n}=1 \\
& \exists n \text { s.t. } \mathbf{M}_{p, j, n}=1 \wedge \mathbf{M}_{j, i, n}=1 \wedge \mathbf{M}_{p, i, n}=0 \\
& \wedge \max \left(\mathbf{t}_{j, n}^{\mathrm{Tx}}\right)>\min \left(\mathbf{t}_{p, n}^{\mathrm{Tx}}\right) .
\end{aligned}
$$

The solution can be obtained via branch-and-bound, which completes in polynomial time on average [7].

Constraint (1b) prevents transmissions on technology $n$ if node $i$ does not have it. Constraint (1c) allows simultaneous transmissions by two nodes $i$ and $j$ in the same slot using technology $n$ only if: $\forall p \neq i, j$, the links $i \leftrightarrow p$ and $j \leftrightarrow p$ do not exist for technology $n$; or if one of the two links exists and $p$ does not transmit in the same slot (lest $p$ would be deaf to $i$ 's or $j$ 's transmission); or otherwise, if both links exist, $p$ also transmits in the same slot (so that $i$ and $j$ 's transmissions would not collide at $p$ ). Constraint (1d) specifies that more slots are given to nodes with more neighbors, so that the total number of slots per neighbor is at least $c_{i} \geq C$. Constraint (1e) imposes that node $i$ be the only transmitter in at least one slot $t$ over each technology $n$. Hence, although (1c) allows primary conflicts, there exists at least one slot for each node to transmit free from interference. Finally, constraint (1f) facilitates that the same packet can propagate further than one hop within the same frame, and is achieved by allowing a node $j$ located at an intermediate position between two nodes $p$ and $i$ to have at least one transmission slot later than node $p$ 's slot, i.e., $\max \left(\mathbf{t}_{j, n}^{\mathrm{Tx}}\right)>\min \left(\mathbf{t}_{p, n}^{\mathrm{Tx}}\right)$.

The formalization in (1) shows that OMS optimizes link utilization by allocating the data flows across all PHYs of a node, while considering possible bottlenecks and packet delays due to the different capabilities of the various PHYs. However, OMS is a centralized solution and thus fits the case of small networks. Still, by sharing the adjacency matrix M, OMS avoids the use of a centralized hub. 
TABLE I

SiMULATIONS: CHARACTERISTICS OF THE PHY TECHNOLOGIES

\begin{tabular}{llll} 
Technology & Bit rate $[\mathrm{bps}]$ & Max range $[\mathrm{m}]$ & Band $[\mathrm{kHz}]$ \\
\hline LF acoustics & 3000 & 4000 & $7-17$ \\
MF acoustics & 6000 & 1500 & $18-34$ \\
HF acoustics & 15000 & 500 & $48-78$ \\
\hline
\end{tabular}

\section{Simulation Results}

Unlike many other scheduling solutions [2], OMS focuses on managing transmissions effectively through diverse PHYs. We therefore compare the performance of OMS with the only two benchmark schemes we found to be appropriate for multimodal underwater scheduling: the Aloha protocol [8, Section 4.2], where a packet is sent as soon as it becomes available (except that no transmission can start if a reception is in progress, in order to replicate the behavior of actual acoustic transceivers); and the TDMA scheme in [4]. In both cases, to transmit on a given link, a node employs the PHY providing the highest bit rate, among those integrated by both itself and the receiver. We consider the packet delivery ratio (PDR),

$$
P D R=N^{r x} / N^{t x},
$$

where $N^{t x}$ and $N^{r x}$ are the total number of packets transmitted and received, respectively; the packet delivery delay (PDD) that is calculated as the time elapsed from the packet generation until its reception; and the service fairness, which we define by Jain's fairness index [9] for the PDR,

$$
\left.J=\left(\sum_{i=1}^{N} P D R_{i}\right)^{2}\left(N \cdot \sum_{i=1}^{N} P D R_{i}\right)^{2}\right)^{-1},
$$

where $P D R_{i}=N_{i}^{r x}\left(\sum_{i=1}^{N} N_{k, i}^{t x}\right)^{-1}$ is the PDR of the packets received by node $i$, and $N_{k, i}^{t x}$ is the number of packets transmitted by node $k$ to node $i$. For $L_{p}$ bits in a packet, we also consider the network throughput

$$
T H R=N^{r x} \cdot L_{p} / T_{s},
$$

where $T_{S}$ is the duration of the simulation.

We deploy a multimodal network of 4 nodes uniformly at random over an area of $2 \times 2 \mathrm{~km}^{2}$ and depth $100 \mathrm{~m}$. We consider three PHYs, based on low-, mid- and highfrequency acoustics (respectively LF, MF and HF for short). The PHY characteristics are summarized in Table I. Due to the random deployment, the MF and HF modems may not form fully connected subnetworks. Every node incorporates an LF modem. At random, two nodes also have an MF modem, and three nodes have an HF modem. We perform a Monte-Carlo set of 600 runs, each with a different random topology realization, using DESERT Underwater [10]. We set $L_{p}=1000$ bytes, and the number of slots $N=12$. Guard times have been chosen according to the propagation time and the bit rate of each PHY.

In Fig. 1, we show the cumulative distribution function (CDF) of the throughput for each technology and for their combination ("Total"). The poor performance of TDMA proves that it is unable to exploit all technologies. Since OMS optimally utilizes all available links, in total its throughput performance always exceeds that of Aloha, with a gain of roughly $100 \%$ in more than $50 \%$ of the cases. We

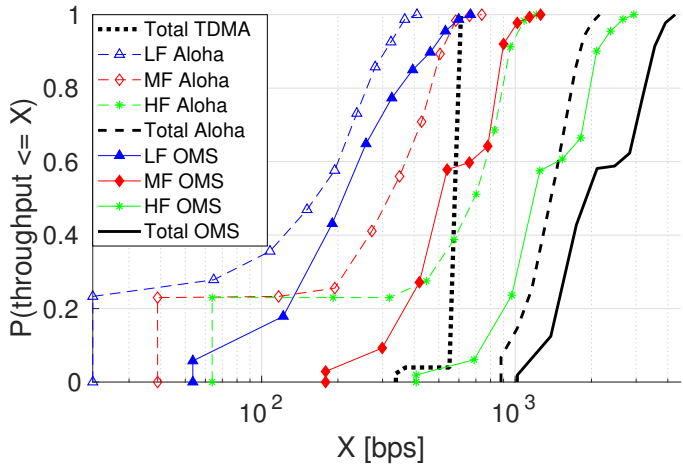

Fig. 1. CDF of the throughput (4) for OMS and Aloha.

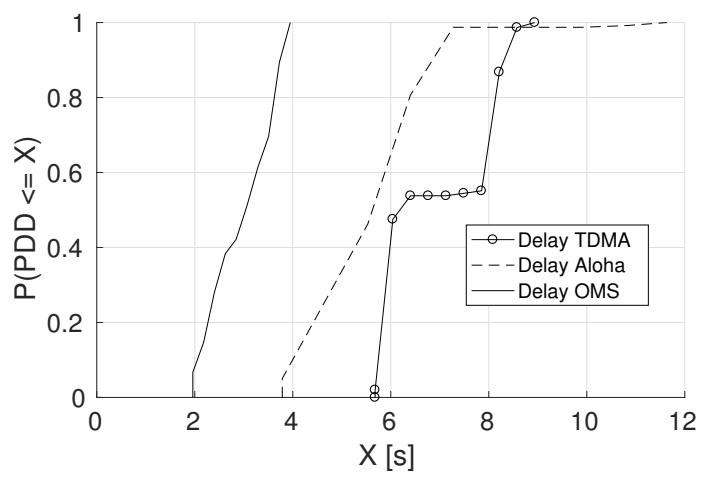

Fig. 2. CDF of packet delivery delay for OMS and Aloha. Total case.

also observe that the gain increases with frequency, since high frequency translates into a higher bit rate, and the link utilization becomes more effective.

From the CDF of the PDD (Fig. 2), we observe that OMS outperforms both Aloha and TDMA by a significant figure of $3 \mathrm{~s}$ and $4 \mathrm{~s}$, respectively, providing a delay that is 50\% and 66\% lower than the other two MAC schemes. Moreover, OMS proves less sensitive to specific topologies than Aloha and TDMA. This is due to constraint (1d), that enforces interference-free slots for all nodes.

\section{SEA EXPERIMENT}

We demonstrated OMS in a sea experiment on May 2017 in Hadera, Israel. The deployment (see Fig. 3) involved four stations: nodes 2 and 4 lowered from a pier stretching $2 \mathrm{~km}$ eastwards from the shore, and nodes 1 and 3 placed on boats. The water depth was $25 \mathrm{~m}$.

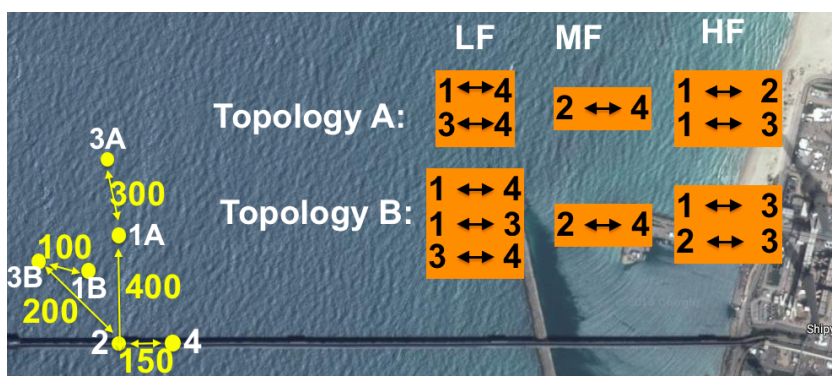

Fig. 3. Sketch of the network deployment in Hadera, Israel. 


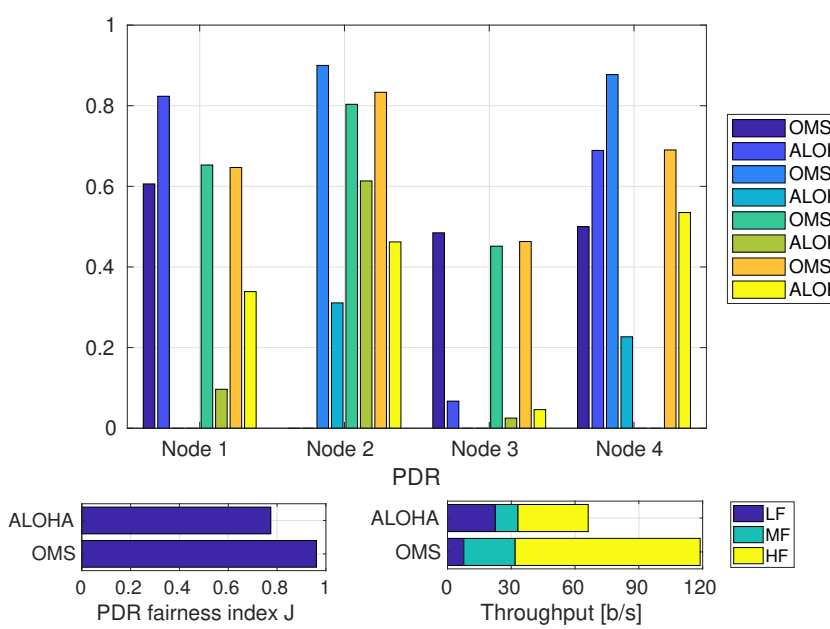

Fig. 4. Topology A: PDR (2), PDR fairness (3), and throughput (4).

We used EvoLogics underwater modems operating in three frequency bands: $7-17 \mathrm{kHz}$ (LF, up to $6.9 \mathrm{kbit} / \mathrm{s}$ ), $18-34 \mathrm{kHz}$ (MF, up to $13.9 \mathrm{kbit} / \mathrm{s}$ ), and $48-78 \mathrm{kHz}(\mathrm{HF}$, up to $31.2 \mathrm{kbit} / \mathrm{s})$. Each node lowered its modems to the same depth, and connected to them via Ethernet from a single laptop. A sea state of 3 resulted in a low PDR.

By changing the locations of nodes 1 and 3 , we tested two network topologies, each for a total of $20 \mathrm{~min}$. To achieve intense network traffic, we let each node transmit a packet whenever possible. Considering the poor performance of TDMA in the simulations, we only focused on the OMS and Aloha protocols in the experiment.

In Fig. 4, we show the PDR, fairness, and throughput performance for Topology A. The differences between nodes are mostly due to the sparse topology, where nodes have a different number of one-hop neighbors. We observe that, except for the LF case, OMS's PDR is consistently better than Aloha's. Similarly, the bottom panels of Fig. 4 show that OMS's transmission fairness and throughput are also better. We note that, due to its channel utilization, the experimental results of OMS also exceed those of an ideal (theoretical) TDMA with perfect PDR.

Fig. 5 shows the performance for Topology B. Compared to Topology A, more LF links are available. This diversity is utilized by OMS. Thus, unlike in Fig. 4, here the PDR of OMS is better than Aloha's also for the LF case. While the fairness performance follows the same trend, OMS's throughput gain decreases. This is mostly because in Topology A there are fewer connection possibilities. Hence, Aloha experienced more collisions than in Topology B. Still, relative to Aloha, in the sea experiment OMS demonstrated a significant performance gain in all metrics.

Based on the data sheets of the manufacturer, the transmission power of the LF, MF, and HF modems is $P_{t x, L F}=40 \mathrm{~W}, P_{t x, M F}=35 \mathrm{~W}$, and $P_{t x, L F}=18 \mathrm{~W}$, respectively. Thus for the transmissions executed during the experiment, where the packet duration was $t=0.4 \mathrm{~s}$, the power consumed for a single packet transmission is $E=$ $t \cdot\left(P_{t x, L F} \cdot N_{t x, L F}+P_{t x, M F} \cdot N_{t x, M F}+P_{t x, H F} \cdot N_{t x, H F}\right) / 3600$.

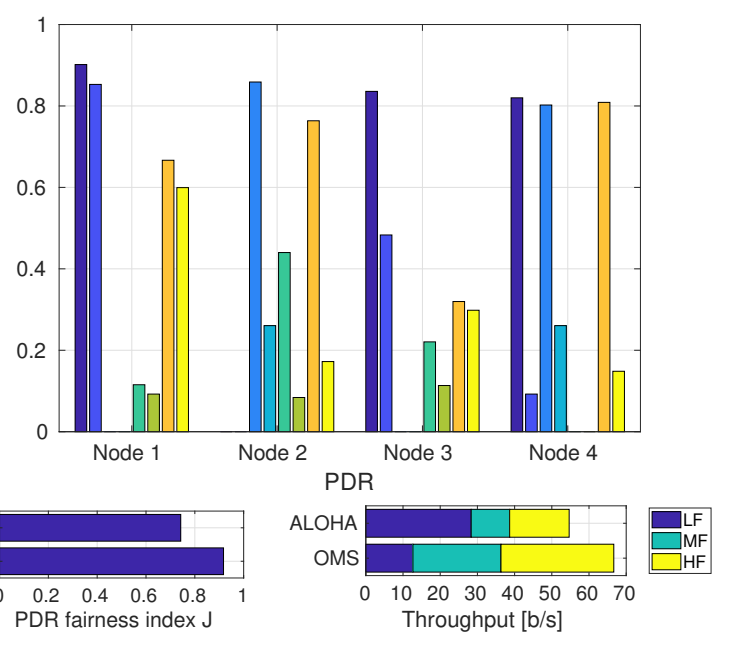

Fig. 5. Topology B: PDR (2), PDR fairness (3), and throughput (4).

Considering the number of packets transmitted, we calculate for Topology 1 a power consumption of $2.2 \mathrm{Wh}$ by OMS and $4.0 \mathrm{~W} / \mathrm{h}$ by Aloha. This power consumption gain of OMS increased for Topology 2, where OMS consumed $2.3 \mathrm{Wh}$, and Aloha consumed 5.1 Wh.

\section{CONCLUSIONS}

We described OMS, a new scheduling protocol for multimodal underwater networks. OMS maximizes the channel utilization while providing a fair quality of service to all nodes, and guaranteeing that at least some of the slots will be free from interference. We have tested OMS both in simulations and in a sea experiment. The results show that OMS fully utilizes the multimodal network, and thus achieves gains in both throughput and packet delivery delay. Future work will include the adaptation of multimodal PHY technologies to network flow requirements.

\section{REFERENCES}

[1] T.-C. Wu et al., "Blue laser diode enables underwater communication at 12.4 Gbps," Nature Scientific reports, vol. 7, Jan. 2017.

[2] V. Gabale et al., "A classification framework for scheduling algorithms in wireless mesh networks," IEEE Commun. Surveys Tuts., vol. 15, no. 1, pp. 199-222, First quarter 2013.

[3] I. Vasilescu et al., "Data collection, storage and retrieval with an underwater optical and acoustical sensor network," in Proc. ACM Sensys, San Diego, CA, Nov. 2005.

[4] F. Campagnaro et al., "On the feasibility of fully wireless remote control for underwater vehicles," in Proc. Asilomar Conf. SS\&C, Pacific Grove, CA, Nov. 2014.

[5] R. Kastner et al., "MURAO: A multi-level routing protocol for acoustic-optical hybrid underwater wireless sensor networks," in Proc. IEEE SECON, Seoul, South Korea, Jun. 2012.

[6] R. Diamant et al., "Topology-efficient discovery: A topology discovery algorithm for underwater acoustic networks," IEEE J. Ocean. Eng., vol. PP, no. 99, pp. 1-15, 2017.

[7] W. Zhang and R. E. Korf, "An average-case analysis of branch-andbound with applications: Summary of results," in Proceedings of the National Conference on AI (AAAI), 1992, pp. 545-550.

[8] A. S. Tanenbaum, Computer Networks. Prentice Hall, 2002.

[9] R. Jain et al., "A quantitative measure of fairness and discrimination for resource allocation in shared computer systems," Tech. Rep. DEC-TR-301, Sep. 1984.

[10] P. Casari et al., "Open-source suites for underwater networking: WOSS and DESERT Underwater," IEEE Network, vol. 28, no. 5, pp. 38-46, Sep. 2014. 